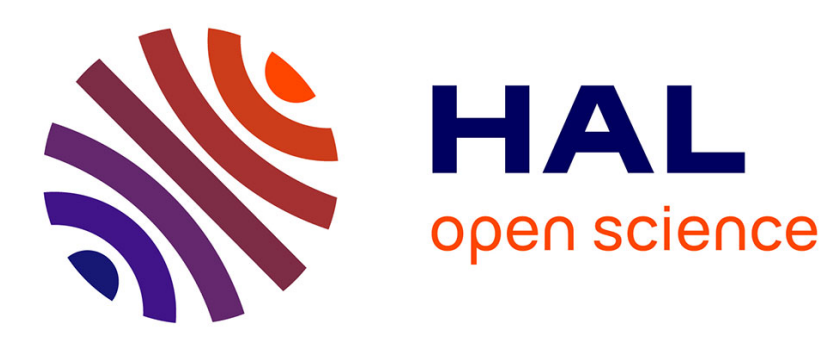

\title{
On the implementation and consequences of the oceanic currents feedback in ocean-atmosphere coupled models
}

\author{
Lionel Renault, Florian Lemarié, Thomas Arsouze
}

\section{To cite this version:}

Lionel Renault, Florian Lemarié, Thomas Arsouze. On the implementation and consequences of the oceanic currents feedback in ocean-atmosphere coupled models. Ocean Modelling, 2019, 141, pp.101423. 10.1016/j.ocemod.2019.101423 . hal-02190847

\section{HAL Id: hal-02190847 \\ https://hal.inria.fr/hal-02190847}

Submitted on 23 Jul 2019

HAL is a multi-disciplinary open access archive for the deposit and dissemination of scientific research documents, whether they are published or not. The documents may come from teaching and research institutions in France or abroad, or from public or private research centers.
L'archive ouverte pluridisciplinaire HAL, est destinée au dépôt et à la diffusion de documents scientifiques de niveau recherche, publiés ou non, émanant des établissements d'enseignement et de recherche français ou étrangers, des laboratoires publics ou privés. 


\title{
On the Implementation and Consequences of the Oceanic Currents Feedback in Ocean-Atmosphere Coupled Models
}

\author{
L. Renault ${ }^{a, *}$, F. Lemarié $^{\mathrm{b}}$, T. Arsouze ${ }^{\mathrm{c}}$ \\ ${ }^{\mathrm{a}}$ LEGOS, University of Toulouse, IRD, CNRS, CNES, UPS, Toulouse, France \\ ${ }^{\mathrm{b}}$ Univ. Grenoble Alpes, Inria, CNRS, Grenoble INP, LJK, Grenoble, France \\ ${ }^{\mathrm{c}}$ Barcelona Supercomputing Center (BSC), Barcelona, Spain
}

\begin{abstract}
The Current FeedBack (CFB) to the atmosphere simply represents the influence of the surface oceanic currents on near-surface wind and surface stress. As the CFB has a significant influence on the oceanic circulation, it is crucial to implement it properly in a coupled Ocean-Atmosphere model. In this study, we first detail the modifications to be implemented into atmospheric models to account for the CFB. In the computation of air-sea fluxes, the relative winds, i.e., the difference between the near-surface winds and the surface oceanic currents, instead of absolute winds have to be used (Modification M1). However, because of the implicit treatment of the bottom boundary condition in most atmospheric models, the use of relative winds also involves a modification of the tridiagonal problem associated with the discretization of the vertical turbulent viscosity (Modification M2). Secondly, we show both analytically and using global coupled simulations that omitting M2 leads to a large underestimation of the surface stress curl response to the CFB and, subsequently, of the coupling coefficient between mesoscale surface stress curl and surface current vorticity. As a consequence, the dampening of the mesoscale activity induced by the CFB is strongly reduced (by a minimum a factor of 2 or more). The practical implementation of the CFB must be done carefully in the atmospheric component of a coupled model in order to avoid a large underestimation of the CFB effect on the oceanic circulation.
\end{abstract}

Key words: Coupled Ocean-Atmosphere Models, Current Feedback to the Atmosphere, Implementation of the Current Feedback in Coupled Models, Consequences of a poor implementation, Oceanic Mesoscale Activity

\footnotetext{
* Corresponding author.

E-mail address: lionel.renaulteird.fr.
} 


\section{Introduction}

We refer to the current feedback (CFB) to the atmosphere as the influence of the oceanic surface currents onto the atmospheric wind and stress. In the past, assuming that the oceanic motions are much weaker than the wind, the CFB has been largely ignored by the atmospheric and oceanic modeling communities. However, numerous studies have shown consequent effects on the oceanic and atmospheric dynamics and thermodynamics. The CFB modifies directly the surface stress (Bye, 1985) and has a "bottom-up" effect on the wind, i.e., a negative stress anomaly causes a positive surface wind anomaly (Renault et al., 2016b, 2019b). A simulation that neglects the CFB do not represent the stress anomalies induced by the CFB as observed by satellite (Renault et al., 2017b, 2019b). From an atmospheric perspective, the CFB leads to small changes in the mean momentum and heat fluxes (1 to 7\%, Duhaut and Straub 2006), with the exception of regions characterized with strong currents and relatively weaks wind such as, e.g., the Equatorial band where the CFB can reduce the median wind stress magnitude by $20 \%$ (Kelly et al., 2001). At the mesoscale, the CFB mainly affects the stress/wind curl but does not have a systematic effect on its magnitude nor divergence (O’Neill et al., 2003; Chelton et al., 2004; Renault et al., 2016b). Both wind and stress response to the $\mathrm{CFB}$ are generally relatively weak with respect to their absolute values (except potentially over regions with strong currents, e.g., tidal currents, and weak winds). For instance a surface current of $1 \mathrm{~m} \mathrm{~s}^{-1}$ causes a $10-\mathrm{m}$ wind anomaly of $\approx 0.3 \mathrm{~m} \mathrm{~s}^{-1}$ (see Renault et al. 2019b). However, because these anomalies are collocated over the eddies and are systematic, they play an important role in the determination of the transfer of energy between the ocean and the atmosphere and in the determination of the ocean dynamic (Renault et al., 2019b). From the oceanic point of view, the CFB leads to a slow down of the mean oceanic circulation (Pacanowski, 1987, Luo et al., 2005) and to a dampening of the oceanic mesoscale and submesoscale activity by inducing sinks of energy from the currents to the atmosphere and a subsequent "eddy killing" process (Dewar and Flierl, 1987; Duhaut and Straub, 2006; Dawe and Thompson, 2006; Eden and Dietze, 2009; Xu and Scott, 2008; Seo et al., 2016; Renault et al., 2016b, 2017a; Oerder et al., 2018; Renault et al., 2018). The CFB also partly controls the Western Boundary Currents by reducing the eddy-mean flow interaction (Renault et al., 2016a, 2017b, 2019a). Finally, by altering the mean oceanic circulation and the sea surface temperature, the CFB can indirectly change the precipitation rate (Renault et al., 2017b). Hence, it seems important to take into account properly the CFB in a coupled model. In this regard, the objective of this study is twofold. The first aim is to describe the necessary modifications that have to be done in the atmospheric models to accurately resolve the CFB. This implies modifications in the implementation of the bulk formula (M1) but also, in some cases, in the tridiagonal problem associated with the implicit treatment of vertical turbulent mixing (M2). M1 is necessary to properly account for the CFB effect on wind-stress norm while M2 is necessary to properly compute the modification of wind-stress orientation by CFB effect. Then, this study strives to characterize the theoretical and practical consequences of a misrepresentation of the CFB in an atmospheric model by applying a simple approach based on a Taylor expansion, but also by using a global high-resolution ocean-atmosphere coupled model. The main focus is on the sensitivity of the stress response to the CFB implementation in coupled oceanatmosphere models. The modifications that have to be done in an atmospheric model are described and discussed in Sec. 2. Sections 3 and 4 aim to characterize the sensitivity of the CFB surface stress response to the modifications that have to be implemented in an atmospheric model, from analytical and global coupled models perspectives, respectively. The results are then discussed and summarized in Sec. 5. 


\section{Implementing the Current Feedback in a Coupled Model}

In this section, we discuss the practical implementation of the oceanic current feedback within a coupled model. As described below, such implementation differs depending on how the boundary condition at the air-sea interface for the vertical turbulent viscosity term is discretized in time in the atmospheric model.

\section{$2.1 \quad$ Surface-layer/atmosphere coupling}

Let us consider the following diffusion equation for an air-column

$$
\partial_{t} \mathbf{u}_{\mathrm{a}}=\partial_{z}\left(\nu \partial_{z} \mathbf{u}_{\mathrm{a}}\right)
$$

with $\mathbf{u}_{\mathrm{a}}$ the horizontal atmospheric velocity vector, $\nu$ the turbulent viscosity given by an adequate boundary layer parameterization scheme and $z$ the vertical direction. In numerical models, the vertical turbulent viscosity is systematically treated implicitly in time for stability reasons due to the small vertical grid distance (relative to the horizontal one) typically used in most configurations. When the CFB is accounted for, the boundary condition for (2.1) at the air-sea interface is

$$
\rho_{a} \nu \partial_{z} \mathbf{u}_{\mathrm{a}}(z=0)=\boldsymbol{\tau}=\rho_{a} C_{D}\left\|\mathbf{u}_{\mathrm{a}}^{0}-\mathbf{u}_{\mathrm{o}}^{0}\right\|\left(\mathbf{u}_{\mathrm{a}}^{0}-\mathbf{u}_{\mathrm{o}}^{0}\right)
$$

with $\boldsymbol{\tau}$ the surface wind-stress, $\mathbf{u}_{\mathrm{o}}^{0}$ the surface oceanic currents, $\mathbf{u}_{\mathrm{a}}^{0}$ the surface winds and $C_{D}$ a drag coefficient provided by a surface-layer parameterization (a.k.a. bulk formulation) and computed using $\mathbf{u}_{\mathrm{o}}^{0}$. Since (2.1) is treated implicitly in time, an estimate of $\boldsymbol{\tau}^{n+1}$ at time $t_{n+1}=(n+1) \Delta t$ is required to advance from $t_{n}$ to $t_{n+1}$ with $\Delta t$ the time-step for the temporal discretization. At this point we can distinguish two possible configurations both leading to a consistent discretization of the stress boundary condition:

(i) The momentum flux computation is done outside the atmospheric model (e.g. in the coupler), in this case the wind-stress would necessarily be

$$
\boldsymbol{\tau}^{n+1}=\rho_{a} C_{D}\left\|\left(\mathbf{u}_{\mathrm{a}}^{0}\right)^{n}-\widetilde{\mathbf{u}}_{\mathrm{o}}^{0}\right\|\left(\left(\mathbf{u}_{\mathrm{a}}^{0}\right)^{n}-\widetilde{\mathbf{u}}_{\mathrm{o}}^{0}\right)
$$

since no information at time $n+1$ is available. This case is often referred to as explicit flux coupling and is subject to a stability constraint (Lemarié et al., 2015; Beljaars et al., 2017). In (2.2) $\widetilde{\mathbf{u}}_{\mathrm{o}}^{0}$ can either represent an instantaneous value at time $n$ or a temporal average depending on the ocean-atmosphere coupling algorithm.

(ii) The momentum flux is computed inside the atmospheric model making it possible to treat the surface boundary condition implicitly in time. We would thus consider that

$$
\boldsymbol{\tau}^{n+1}=\rho_{a} C_{D}\left\|\left(\mathbf{u}_{\mathrm{a}}^{0}\right)^{n}-\widetilde{\mathbf{u}}_{\mathrm{o}}^{0}\right\|\left(\left(\mathbf{u}_{\mathrm{a}}^{0}\right)^{n+1}-\widetilde{\mathbf{u}}_{\mathrm{o}}^{0}\right)
$$

This case is often referred to as implicit flux coupling and is expected not to raise any stability issue (Beljaars et al. 2017, their App. B). Since (2.3) involves $\left(\mathbf{u}_{\mathrm{a}}^{0}\right)^{n+1}$ it requires adjustments in the tridiagonal matrix associated with the implicit treatment of (2.1). Note that the numerically correct boundary condition $\boldsymbol{\tau}^{n+1}=\rho_{a} C_{D}\left\|\left(\mathbf{u}_{\mathrm{a}}^{0}\right)^{n+1}-\widetilde{\mathbf{u}}_{\mathrm{o}}^{0}\right\|\left(\left(\mathbf{u}_{\mathrm{a}}^{0}\right)^{n+1}-\widetilde{\mathbf{u}}_{\mathrm{o}}^{0}\right)$ is never used in practice in numerical models because it would require the solution of an iterative problem. This is the reason why the linearized boundary condition (2.3) is considered here. 


\subsection{Implications for CFB implementation}

In the case of an explicit flux coupling, adding the oceanic currents feedback does not require any modification of the atmospheric and oceanic codes. It is only necessary to replace the absolute wind $\mathbf{u}_{\mathrm{a}}^{0}$ by the relative wind $\mathbf{u}_{\mathrm{a}}^{0}-\mathbf{u}_{\mathrm{o}}^{0}$ within the surface layer parameterization to compute $C_{D}$ and when computing the wind stress. When an implicit flux coupling is used, the same modification is required in the surfacelayer parameterization (M1) but it is also needed to adjust the implicit treatment of the vertical viscous term (M2). Indeed, with boundary condition (2.3) the discretization of (2.1) in the bottom most grid cell (with index $k=1$, i.e. $\left.\left(\mathbf{u}_{\mathrm{a}}\right)_{k=1} \equiv \mathbf{u}_{\mathrm{a}}^{0}\right)$ ) in the atmospheric model would be

$$
\begin{array}{r}
\left(1+\frac{\nu_{3 / 2} \Delta t}{\Delta z_{1} \Delta z_{3 / 2}}+\frac{C_{D} \Delta t}{\Delta z_{1}}\left\|\left(\mathbf{u}_{\mathrm{a}}\right)_{k=1}^{n}-\widetilde{\mathbf{u}}_{\mathrm{o}}^{0}\right\|\right)\left(\mathbf{u}_{\mathrm{a}}\right)_{k=1}^{n+1}-\left(\frac{\nu_{3 / 2} \Delta t}{\Delta z_{1} \Delta z_{3 / 2}}\right)\left(\mathbf{u}_{\mathrm{a}}\right)_{k=2}^{n+1} \\
=\left(\mathbf{u}_{\mathrm{a}}\right)_{k=1}^{n}+\frac{C_{D} \Delta t}{\Delta z_{1}}\left\|\left(\mathbf{u}_{\mathrm{a}}\right)_{k=1}^{n}-\widetilde{\mathbf{u}}_{\mathrm{o}}^{0}\right\| \widetilde{\mathbf{u}}_{\mathrm{o}}^{0}
\end{array}
$$

where $\Delta z_{1}$ is the thickness of the grid cell $k=1, \Delta z_{3 / 2}$ the distance between the centers of grid cells $k=1$ and $k=2$ and $\nu_{3 / 2}$ the turbulent viscosity at the interface between grid cells $k=1$ and $k=2$ (see Lemarié (2015) for all the details on the derivation of (2.4)). In (2.4), the second term in the right-hand-side is non-zero only when CFB is included. Omitting this term (i.e. omitting modification M2) would amount to consider an incorrect boundary condition

$$
\boldsymbol{\tau}^{n+1}=\rho_{a} C_{D}\left\|\left(\mathbf{u}_{\mathrm{a}}^{0}\right)^{n}-\widetilde{\mathbf{u}}_{\mathrm{o}}^{0}\right\|\left(\mathbf{u}_{\mathrm{a}}^{0}\right)^{n+1}
$$

As far as the oceanic model is concerned, the CFB on the wind-stress does not require any modifications besides providing $\mathbf{u}_{\mathrm{o}}^{0}$ to the coupling interface. Unlike discretizations (2.2) and (2.3), discretization (2.5) is inconsistent in the sense that for $\Delta t \rightarrow 0$ it does not converge toward the correct continuous form of the bottom boundary condition. Since the majority of atmospheric models uses an implicit flux coupling, we study the consequences of an incomplete implementation of the CFB effect in this case in the next section.

\section{Analytical Analysis}

In the following we assume that the sensitivity of the drag coefficient $C_{D}$ to the CFB is negligibly weak and that $\Delta t$ is sufficiently small to consider that $\left\|\mathbf{u}_{\mathrm{a}}^{0}-\mathbf{u}_{\mathrm{o}}^{0}\right\|$ and $\left(\mathbf{u}_{\mathrm{a}}^{0}-\mathbf{u}_{\mathrm{o}}^{0}\right)$ are evaluated at the same moment in time. Doing so, we can express the wind-stress using the generic formulation

$$
\boldsymbol{\tau}_{\alpha, \lambda}=\rho_{a} C_{D}\left\|\mathbf{u}_{\mathrm{a}}^{0}-\alpha \mathbf{u}_{\mathrm{o}}^{0}\right\|\left(\mathbf{u}_{\mathrm{a}}^{0}-\lambda \mathbf{u}_{\mathrm{o}}^{0}\right)
$$

When the CFB is ignored we simply have $(\alpha, \lambda)=(0,0)$ while when the CFB is accounted for we have $(\alpha, \lambda)=(1,1)$. In the event the CFB is improperly implemented by omitting the second term in the rhs in (2.4) we would have $(\alpha, \lambda)=(1,0)$. Considering that $\left\|\mathbf{u}_{\mathrm{o}}^{0}\right\| \ll\left\|\mathbf{u}_{\mathrm{a}}^{0}\right\|$ we easily obtain

$$
\left\|\mathbf{u}_{\mathrm{a}}^{0}-\mathbf{u}_{\mathrm{o}}^{0}\right\| \approx\left\|\mathbf{u}_{\mathrm{a}}^{0}\right\|-\mathbf{e}_{a} \cdot \mathbf{u}_{\mathrm{o}}^{0}+\mathscr{O}\left(\left(u_{\mathrm{o}}^{0}\right)^{2},\left(v_{\mathrm{o}}^{0}\right)^{2}, u_{\mathrm{o}}^{0} v_{\mathrm{o}}^{0}\right), \quad \mathbf{e}_{\mathrm{a}}=\frac{\mathbf{u}_{\mathrm{a}}^{0}}{\left\|\mathbf{u}_{\mathrm{a}}^{0}\right\|}
$$

where $\mathbf{e}_{a}$ is a unit vector in the wind direction and $\mathbf{u}_{\mathrm{o}}^{0}=\left(u_{\mathrm{o}}^{0}, v_{\mathrm{o}}^{0}\right)$. In the spirit of Bye (1985); Rooth and Xie (1992); Duhaut and Straub (2006); Gaube et al. (2015) or Renault et al. (2017a), using this Taylor expansion we can express $\boldsymbol{\tau}_{\alpha, \lambda}^{\prime}$ the stress difference between $\boldsymbol{\tau}_{\alpha, \lambda}$ and $\boldsymbol{\tau}_{0,0}$ as 

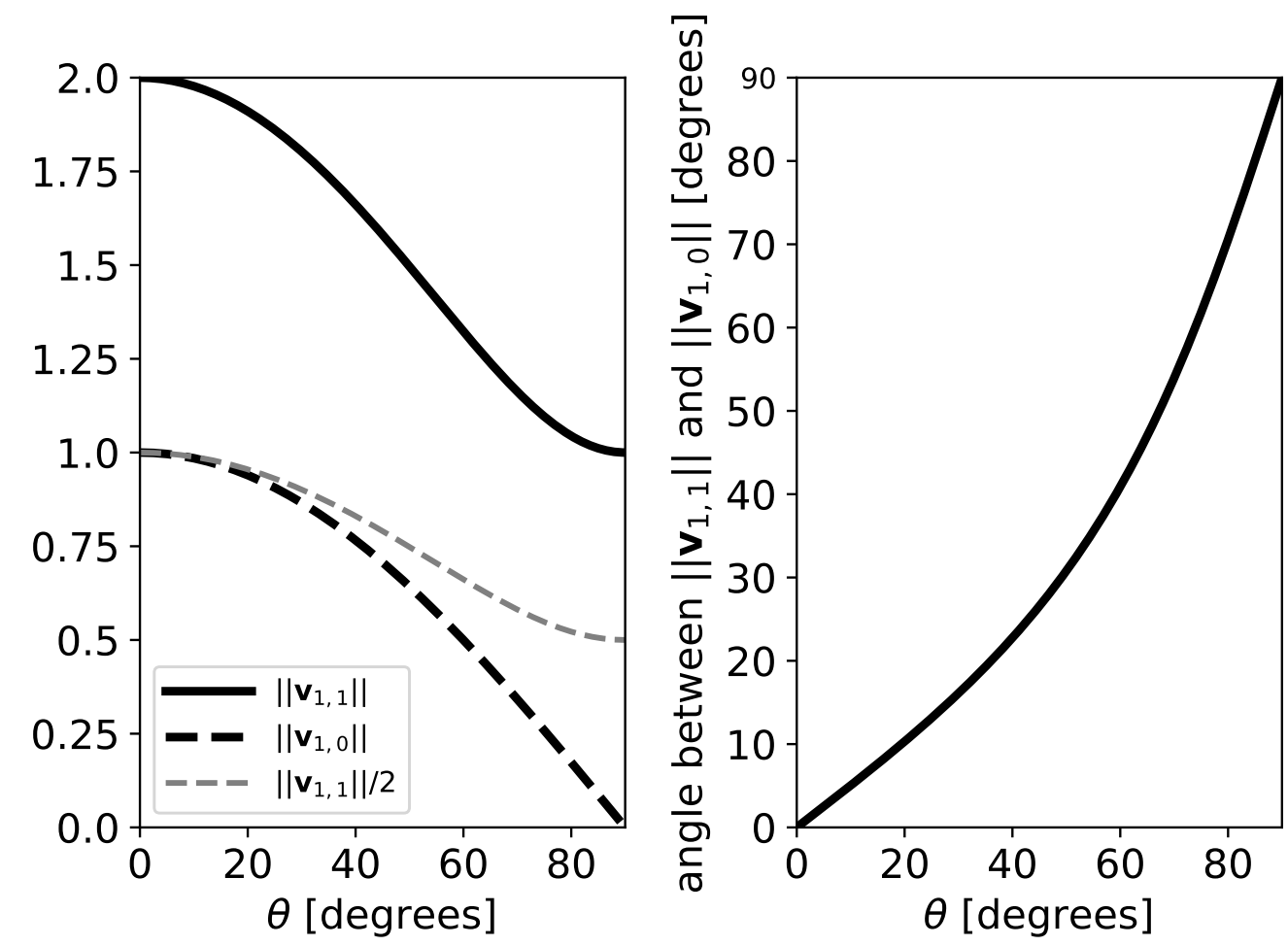

Fig. 1. Norm of the vectors $\mathbf{v}_{1,1}=(2 \cos \theta, \sin \theta)$ and $\mathbf{v}_{1,0}=(\cos \theta, 0)$ with respect to the angle $\theta$ between the surface winds and oceanic currents (left). Angle between $\mathbf{v}_{1,1}$ and $\mathbf{v}_{1,0}$ computed as $\arccos \left(\frac{2|\cos \theta|}{\sqrt{(2 \cos \theta)^{2}+(\sin \theta)^{2}}}\right)$ (bottom).

$$
\begin{aligned}
\boldsymbol{\tau}_{\alpha, \lambda}^{\prime} & \approx-\rho_{a} C_{D}\left[\lambda\left\|\mathbf{u}_{\mathrm{a}}^{0}\right\| \mathbf{u}_{\mathrm{o}}^{0}+\alpha\left(\mathbf{e}_{\mathrm{a}} \cdot \mathbf{u}_{\mathrm{o}}^{0}\right) \mathbf{u}_{\mathrm{a}}^{0}\right] \\
& \approx-\rho_{a} C_{D}\left\|\mathbf{u}_{\mathrm{a}}^{0}\right\|\left\|\mathbf{u}_{\mathrm{o}}^{0}\right\|\left\{\lambda \mathbf{e}_{\mathrm{o}}+\alpha\left(\mathbf{e}_{\mathrm{a}} \cdot \mathbf{e}_{\mathrm{o}}\right) \mathbf{e}_{\mathrm{a}}\right\}
\end{aligned}
$$

with $\mathbf{e}_{o}$ the unit vector in the oceanic surface currents direction. First we can notice that when the winds and the currents are aligned we exactly have $\tau_{1,1}^{\prime}=2 \tau_{1,0}^{\prime}$. If we express all the vectors in the orthonormal basis formed by the two vectors $\mathbf{e}_{1}=(1,0)$ and $\mathbf{e}_{2}=(0,1)$ such that $\mathbf{e}_{1}=\mathbf{e}_{a}$, the term in curly bracket in (3.2) is equal to $\mathbf{v}_{\alpha, \lambda}^{\prime}=((\alpha+\lambda) \cos \theta, \lambda \sin \theta)$ with $\theta$ the angle between the winds and the currents. In particular we would have $\mathbf{v}_{1,1}^{\prime}=(2 \cos \theta, \sin \theta)$ and $\mathbf{v}_{1,0}^{\prime}=(\cos \theta, 0)$. In Fig. 1, the norms $\left\|\mathbf{v}_{1,1}^{\prime}\right\|$ and $\left\|\mathbf{v}_{1,0}^{\prime}\right\|$ as well as the angle between $\mathbf{v}_{1,1}^{\prime}$ and $\mathbf{v}_{1,0}^{\prime}$ are represented as functions of the angle $\theta$ between the winds and the oceanic currents. It can readily be seen that by omitting the second term in the rhs in (2.4), the intensity of the stress anomalies induced by the CFB are divided by at least two and even more than two when the angle $\theta$ between the surface winds and currents is larger than $45^{\circ}$. The orientation of the stress anomaly is also largely incorrect (Fig 1, right panel). Using $\boldsymbol{\tau}_{1,0}$ instead of $\boldsymbol{\tau}_{1,1}$ largely underestimates the CFB effect on the stress curl, and, thus, the mesoscale activity dampening (see next section). However, this simple analytical development does not consider the wind response to the CFB that will partly counteract the stress response (Renault et al., 2016b). Additionally, as shown by Pelletier et al. (2018), $C_{D}$ can also be impacted by the CFB, but its response strongly depends on the bulk formula used. 


\section{Characterizing the Surface Stress Response to the Current Feedback in Coupled Simulations}

\subsection{Model Configuration}

EC-Earth is a global coupled climate model (Hazeleger et al., 2012) that has been developed by a consortium of European institutes that consist presently of 27 research institutes (http://www. ec-earth. org/). It is used in a wide range of studies spanning in time from paleoresearch to climate projections, including also seasonal and decadal forecasts. The atmospheric component of EC-Earth is the Integrated Forecast System (IFS), based on version cycle-36r4 of the European Centre for Medium Range Weather Forecasts (ECMWF). It uses a reduced Gaussian-grid with 91 vertical levels. The horizontal domain is based on a spectral truncation at T1279 (corresponding to a nominal spatial resolution of $\approx 15 \mathrm{~km}$ globally). The H-TESSEL model is used for the land surface and is an integral part of the IFS atmospheric model, for more details see Hazeleger et al. (2010).

The ocean component is the Nucleus for European Modelling of the Ocean (NEMO, Madec et al. 2015) version 3.6. It uses a tri-polar grid with poles over northern North America, Siberia and Antarctica with a resolution of about $\frac{1}{12}^{\circ}$ (the so-called ORCA12 configuration) and 75 vertical levels. NEMO includes the Louvain la Neuve sea-ice model version 3 (LIM3, Vancoppenolle et al. 2012), which is a dynamic thermodynamic sea-ice model with 5 ice thickness categories. The atmosphere/land and ocean/sea ice components are coupled through the OASIS-MCT (Ocean, Atmosphere, Sea Ice, Soil) coupler (Craig et al., 2017) at hourly frequency.

\subsection{Coupled Simulations}

A set of three global coupled simulations is performed with the setup described in Sec. 4.1 over a period of 1-day. As explained hereafter, this time period is long-enough to illustrate the stress response to the CFB and its sensitivity to the modifications of the bulk formula (M1) and of the tridiagonal problem associated with the discretization of the vertical turbulent mixing (M2) described in Sec. 2. However, to verify it, the simulations have also been run for a period of 1-month. The coupled simulations only differ by the degree of coupling they consider. NOCRT does not consider the CFB and, thus, uses the absolute wind to estimate the surface stress (see Sec. 2 and Eq. 3.1 with $(\alpha, \lambda)=(0,0)$ ). $C R T_{10}$ considers only M1, omitting M2 $((\alpha, \lambda)=(1,0))$, whereas $C R T_{11}$ considers both $\mathbf{M} 1$ and $\mathbf{M} 2((\alpha, \lambda)=(1,1))$. The three simulations start from the same restart after a 10-years spin up that uses the NOCRT configuration. Note the state of the ocean in the restart does not really matter here because only the stress response to the CFB is assessed and not directly the oceanic response.

\subsection{Mesoscale Stress Response to the CFB}

As shown in Sec. 2 and 3, the CFB instantaneously induces surface stress anomalies that are collocated right over the surface currents and are anti-correlated: e.g., a negative surface current mesoscale anomaly causes a positive surface stress mesoscale anomaly (and vice versa). The wind response is mainly situated at the near-surface (Renault et al. 2016b). Its adjustment time corresponds to the adjustment time of the Planetary Boundary Layer (PBL) to a change in the surface boundary condition, which is roughly 1 hour. The wind response only partially counteracts the surface stress changes: e.g., a decrease of the surface stress causes an increase of the wind (Renault et al., 2016b). At the mesoscale, the stress response to the 
CFB is usually assessed using its curl because mesoscale currents are very nearly geostrophic and mainly non-divergent (O'Neill et al., 2003; Chelton et al., 2004, Renault et al., 2016b) and because it allows to properly characterize the stress response (Renault et al., 2017a, 2019b).

The impact of the CFB at the oceanic mesoscale on the surface stress curl in $C R T_{10}$ and $C R T_{11}$ is estimated in two steps. First, the mesoscale surface stress curl and surface current vorticity are estimated for each simulations using a high-pass Gaussian spatial filter with a $250 \mathrm{~km}$ cut-off (as in Renault et al. 2017a). Then, because after one day the atmospheric and oceanic large scale circulations and the sea surface temperature are not significantly different in all the simulations (not shown), the CFB impacts in $C R T_{10}$ and $C R T_{11}$ can simply be isolated by computing the mesoscale surface stress curl difference between these simulations and NOCRT. A one-day simulation has therefore the advantage to have a very low cost in term of computational hours and also to allow to cleanly remove from the surface stress curl both weather related variability and thermal feedback imprint. Figure 2 depicts the mesoscale surface stress curl and surface currents vorticity anomalies as estimated from $C R T_{10}$ and $C R T_{11}$ over a region that encompasses the Gulf Stream. In $C R T_{11}$, where the CFB is fully taken into account, consistent with e.g., Chelton et al. (2001) and Renault et al. (2016a), the surface currents have a clear imprint on the mesoscale surface stress curl over the Gulf Stream path and over mesoscale eddies: a negative current vorticity causes a positive stress curl anomaly. By contrast, in $C R T_{10}$, consistent with Sec. 3 the surface stress curl anomalies are largely reduced because the changes of the surface stress orientation are omitted (Section 2 and 3). Note that the use of a 1-month simulation leads to similar results in term of location and intensity of the induced stress curl anomalies. However, the synoptic variability has to be removed by using a 29-day average and the TFB impact on the stress curl can not be properly removed (Renault et al. 2019b).

\subsection{CFB Coupling Coefficient and Sinks of energy}

At the mesoscale, the surface stress anomalies induced by the CFB cause sinks of energy from the surface currents to the atmosphere, which in turn are responsible of the eddy killing effect in the ocean, i.e., a large dampening of the mesoscale activity (by $\approx 30 \%$ at a quasi-global scale, Renault et al. 2019b; Jullien et al. 2019). As suggested by Renault et al. (2017a), the sinks of energy can be predicted as the product of the coupling coefficient between the mesoscale surface current and the mesoscale surface stress $\left(s_{\tau}\right)$ and the Eddy Kinetic Energy ( $E K E$, a measure of the mesoscale activity). $s_{\tau}$ allows to characterize the efficiency of the eddy killing. The more negative the $s_{\tau}$, the more efficient the eddy killing, and, thus, the larger the dampening of the mesoscale activity. $s_{\tau}$ can therefore be used as a proxy of a correct implementation of the CFB in a coupled model.

As in Renault et al. (2017a) and Renault et al. (2019b), $s_{\tau}$ is estimated as the slope of the linear regression between mesoscale surface stress curl and oceanic current vorticity (estimated following the method described above) and over a given region. $s_{\tau}$ is characterized by spatial and temporal variations that are driven by the magnitude of the near-surface wind (Renault et al., 2017a). Here, $s_{\tau}$ is estimated over the global domain and over the Gulf Stream (Figure 3). In $C R T_{11}$, over the Gulf Stream, $s_{\tau}=-1.4410^{-2}$ $\mathrm{N} \mathrm{s} \mathrm{m}^{-3}$, whereas as the global scale, $s_{\tau}=-0.6510^{-2} \mathrm{~N} \mathrm{~s} \mathrm{~m}^{-3}$, i.e., weaker because the global domain includes many regions of much weaker stress curl and current vorticity mesoscale variability than the Gulf Stream. Note that the absolute values of $s_{\tau}$ estimated here are only valid for this specific day as $s_{\tau}$ is mainly driven by the near-surface wind. From $C R T_{11}$ to $C R T_{10}$, at the global scale and over the Gulf Stream, $s_{\tau}$ is reduced by a factor $\approx 3$ because in $C R T_{10}$ the CFB is improperly implemented as it omits 

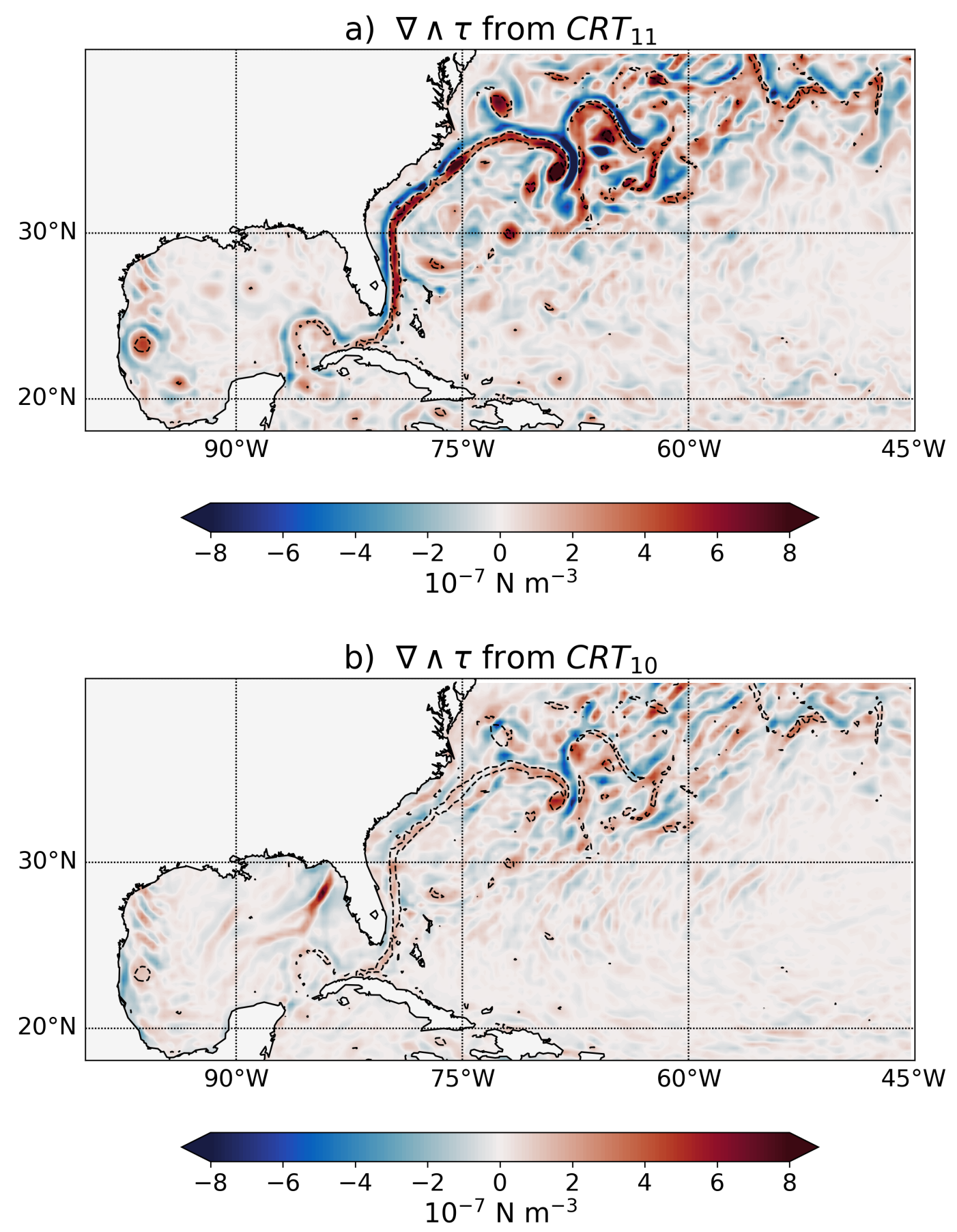

Fig. 2. Snapshot of the Mesoscale surface stress curl induced by the current feedback (CFB) as estimated from (a) $C R T_{11}$ and (b) $C R T_{10}$. The dashed contour-line represents the $-210^{-5} \mathrm{~s}^{-1}$ surface mesoscale current vorticity contour (only one contour is shown for clarity) As expected, the CFB induces mesoscale surface stress curl anomalies collocated over the eddies and the Gulf Stream. In a simulation that ignores the modification M2 (i.e., $C R T_{10}$ ), the mesoscale stress induced anomalies are largely underestimated.

M2 (i.e., $(\alpha, \lambda)=(1,0)$ ), and, thus, the rectification of the stress orientation. Similar results are found for any other regions of the World Ocean (not shown). To verify the validity of the 1-day simulation, $s_{\tau}$ is also estimated using 1-month simulations and using spatial and temporal filter as in Renault et al. (2019b). An underestimation by a factor of at least 2.5 is found, which is consistent with the one-day simulations results. 
a) Global

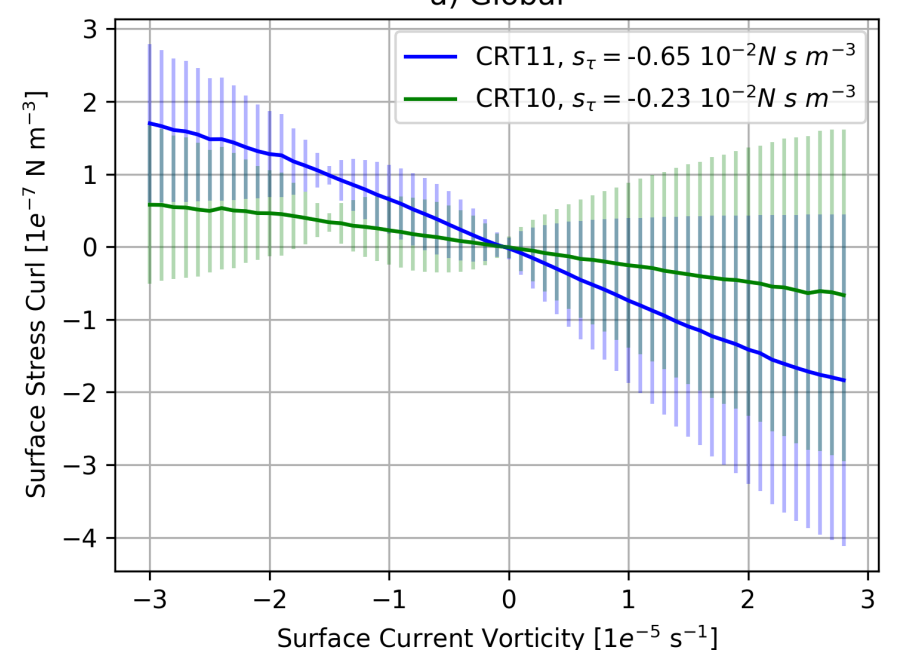

b) Gulf Stream

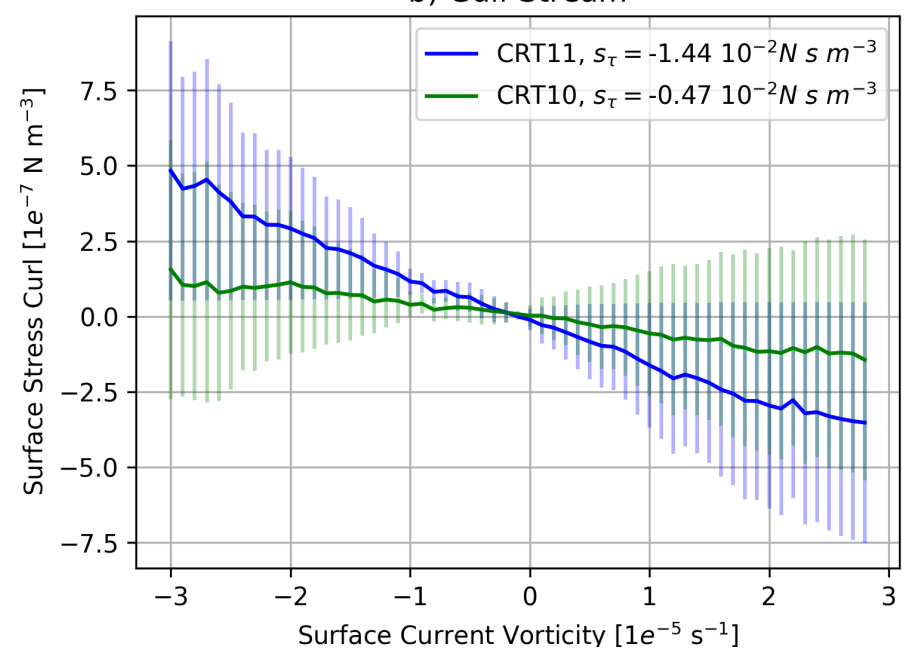

Fig. 3. Linear regression between mesoscale surface current vorticity and surface stress curl as estimated from $C R T_{11}$ and $C R T_{10}$. The error-bars represent the standard deviations around the mean values. The coupling coefficient between mesoscale surface current vorticity and surface stress curl $\left(s_{\tau},\left[\mathrm{N} \mathrm{s} \mathrm{m}^{-3}\right]\right)$ is defined as the slope of the linear regression. (a) At the global scale; (b) over the Gulf Stream. Consistent with Fig. 2, ignoring the modification M2 causes a large underestimation of $s_{\tau}$ everywhere in the World Ocean. A too weak $s_{\tau}$ will result in an underestimation of the dampening of the mesoscale activity induced by the CFB.

The $s_{\tau}$ reduction found from the coupled simulations is also consistent with the analytical prediction presented in the previous section but does take the wind response and the $C_{D}$ variations into account. From an oceanic perspective, the systematic underestimation of $s_{\tau}$ in $C R T_{10}$ will reduce the sinks of energy induced by the CFB (by a factor of at least 2), and, thus, cause an underestimation of the dampening of the mesoscale activity by roughly the same order of magnitude (Renault et al. 2017a).

\section{Conclusion}

The $\mathrm{CFB}$, by causing surface stress anomalies, modulates the oceanic circulation by slowing down the mean oceanic circulation and dampening the mesoscale activity, and by altering the Western Boundary 
Currents. In order to properly account for its effect on the wind-stress magnitude and orientation in most coupled models (i.e., those with an implicit coupling where the wind stress computed within the atmospheric model). We have shown that two modifications are needed both in the bulk formula (Modification M1) but also of the tridiagonal problem associated with the discretization of the vertical turbulent mixing (Modification M2). M1 insures to consider the effect of the CFB on the friction velocity, whereas M2 allows to fully take into account the CFB effect on the surface stress orientation, which is the main effect of the CFB.

By using a simple analytical development of the stress estimation in a bulk formula, we furthermore confirm that ignoring M2 should lead to an underestimation of the stress response to the CFB by a factor of at least 2. However, this simple approach does not take into account, e.g., the surface drag nor the wind responses to the $\mathrm{CFB}$. Using realistic global coupled simulations, we finally show that ignoring M2 actually causes an even larger underestimation of the stress response and of the coupling coefficient $s_{\tau}$ by a factor of $\approx 3 . s_{\tau}$ controls the sinks of energy and the eddy killing process induced by the CFB: the more negative the $s_{\tau}$, the more efficient the eddy killing, and, thus, the dampening of the mesoscale activity. Ignoring M2 can thus lead to an underestimation of the mesoscale activity dampening by factor of $\approx 3$. Note that for coarse resolution models (i.e., models that do not resolve the oceanic mesoscale activity), ignoring M2 should lead to a poor representation of the slow-down of the mean currents induced by the CFB (Luo et al., 2005; Renault et al., 2016a).

To the authors' knowledge, CFB has been incorporated correctly (i.e., both M1 and M2) in IFS (atmospheric model used in Sec. 4) and WRF models (since version WRF3.3.1). In the latter, M2 modification is somehow tedious because it must be done for each PBL parameterization since the building of the tridiagonal system is done locally in the parameterization routines and not through a common interface. Thus, in WRF, the CFB is only taken into account into the Mellor, Yamada,Nakanishi, Niino level 2.5 (MYNN2.5, In the current literature on CFB and based on coupled models, the authors do not systematically specify if CFB is implemented or how the implementation has been done. Considering the importance of the correctness of its implementation in atmospheric models, we recommend this should be specified from now on. Finally, future studies should aim at assessing the sensitivity of the surface stress and wind responses to the use of different PBL schemes.

\section{Acknowledgements}

We appreciate support from the National Science Foundation (OCE-1419450). This work used GENCI and PRACE computing resources in the framework of project 16163939. T.A. and L.R. would like to thank HPC-Europa3 program, application HPC17IUTPN and HPC17MM0RX, respectively, and Barcelona Supercomputing Center (BSC) for hosting them. F. Lemarié acknowledges the support of the French National Research Agency (ANR) through contract ANR-16-CE01-0007. The research leading to these results has received funding from the European Commission's Horizon 2020 project PRIMAVERA (GA 641727).

\section{References}

Beljaars, A., Dutra, E., Balsamo, G., Lemarié, F., 2017. On the numerical stability of surface-atmosphere coupling in weather and climate models. Geosci. Model Dev. 10(2), 977-989. 
Bye, J.A., 1985. Large-scale momentum exchange in the coupled atmosphere-ocean. Elsevier oceanography series 40, 51-61.

Chelton, D.B., Esbensen, S.K., Schlax, M.G., Thum, N., Freilich, M.H., Wentz, F.J., Gentemann, C.L., McPhaden, M.J., Schopf, P.S., 2001. Observations of coupling between surface wind stress and sea surface temperature in the eastern tropical pacific. Journal of Climate 14(7), 1479-1498.

Chelton, D.B., Schlax, M.G., Freilich, M.H., Milliff, R.F., 2004. Satellite measurements reveal persistent small-scale features in ocean winds. science 303(5660), 978-983.

Craig, A., Valcke, S., Coquart, L., 2017. Development and performance of a new version of the oasis coupler, oasis3-mct_3. 0. Geoscientific Model Development 10(9), 3297-3308.

Dawe, J.T., Thompson, L., 2006. Effect of ocean surface currents on wind stress, heat flux, and wind power input to the ocean. Geophysical Research Letters 33(9). L09604.

Dewar, W.K., Flierl, G.R., 1987. Some effects of the wind on rings. Journal of Physical Oceanography 17(10), 1653-1667.

Duhaut, T.H., Straub, D.N., 2006. Wind stress dependence on ocean surface velocity: Implications for mechanical energy input to ocean circulation. Journal of Physical Oceanography 36(2), 202-211.

Eden, C., Dietze, H., 2009. Effects of mesoscale eddy/wind interactions on biological new production and eddy kinetic energy. Journal of Geophysical Research: Oceans 114(C5). C05023.

Gaube, P., Chelton, D.B., Samelson, R.M., Schlax, M.G., ONeill, L.W., 2015. Satellite observations of mesoscale eddy-induced Ekman pumping. Journal of Physical Oceanography 45(1), 104-132.

Hazeleger, W., Severijns, C., Semmler, T., Ştefănescu, S., Yang, S., Wang, X., Wyser, K., Dutra, E., Baldasano, J.M., Bintanja, R., et al., 2010. Ec-earth: a seamless earth-system prediction approach in action. Bulletin of the American Meteorological Society 91(10), 1357-1364.

Hazeleger, W., Wang, X., Severijns, C., Ştefănescu, S., Bintanja, R., Sterl, A., Wyser, K., Semmler, T., Yang, S., Van den Hurk, B., et al., 2012. Ec-earth v2. 2: description and validation of a new seamless earth system prediction model. Climate dynamics 39(11), 2611-2629.

Jullien, S., Masson, S., Colas, F., Oerder, V., Renault, L., 2019. Impact of resolution and current feedback on air-sea energy transfer. To be Submitted to JGR .

Kelly, K.A., Dickinson, S., McPhaden, M.J., Johnson, G.C., 2001. Ocean currents evident in satellite wind data. Geophysical Research Letters 28(12), 2469-2472.

Lemarié, F., 2015. Numerical modification of atmospheric models to include the feedback of oceanic currents on air-sea fluxes in ocean-atmosphere coupled models. Technical Report RT-464, available on https://hal.inria.fr/hal-01184711. Inria Grenoble; Laboratoire Jean Kuntzmann ; Université de GrenobleAlpes ; Inria.

Lemarié, F., Blayo, E., Debreu, L., 2015. Analysis of ocean-atmosphere coupling algorithms: Consistency and stability. Procedia Computer Science 51(Supplement C), 2066 - 2075. International Conference On Computational Science, ICCS 2015.

Luo, J.J., Masson, S., Roeckner, E., Madec, G., Yamagata, T., 2005. Reducing climatology bias in an ocean-atmosphere cgcm with improved coupling physics. Journal of climate 18(13), 2344-2360.

Madec, G., et al., 2015. Nemo ocean engine .

Oerder, V., Colas, F., Echevin, V., Masson, S., Lemarié, F., 2018. Impacts of the mesoscale oceanatmosphere coupling on the peru-chile ocean dynamics: The current-induced wind stress modulation. Journal of Geophysical Research: Oceans .

O’Neill, L.W., Chelton, D.B., Esbensen, S.K., 2003. Observations of sst-induced perturbations of the wind stress field over the southern ocean on seasonal timescales. Journal of Climate 16(14), 2340-2354.

Pacanowski, R., 1987. Effect of equatorial currents on surface stress. Journal of physical oceanography 
17(6), 833-838.

Pelletier, C., Lemarié, F., Blayo, E., 2018. Sensitivity analysis and metamodels for the bulk parametrization of turbulent air-sea fluxes. Quarterly Journal of the Royal Meteorological Society 144(712), 658-669.

Renault, L., Marchesiello, P., Masson, S., McWilliams, J.C., 2019a. Remarkable control of western boundary currents by eddy killing, a mechanical air-sea coupling process. Geophysical Research Letters 46(5), 2743-2751.

Renault, L., Masson, S., Oerder, V., Jullien, S., Colas, F., 2019b. Disentangling the mesoscale oceanatmosphere interactions. Journal of Geophysical Research: Oceans 124(3), 2164-2178.

Renault, L., McWilliams, J.C., Gula, J., 2018. Dampening of submesoscale currents by air-sea stress coupling in the californian upwelling system. Scientific Reports 8(1), 13388.

Renault, L., McWilliams, J.C., Masson, S., 2017a. Satellite observations of imprint of oceanic current on wind stress by air-sea coupling. Scientific Reports 7(1), 17747.

Renault, L., McWilliams, J.C., Penven, P., 2017b. Modulation of the agulhas current retroflection and leakage by oceanic current interaction with the atmosphere in coupled simulations. Journal of Physical Oceanography 47(8), 2077-2100.

Renault, L., Molemaker, M.J., Gula, J., Masson, S., McWilliams, J.C., 2016a. Control and stabilization of the gulf stream by oceanic current interaction with the atmosphere. Journal of Physical Oceanography 46(11), 3439-3453.

Renault, L., Molemaker, M.J., Mcwilliams, J.C., Shchepetkin, A.F., Lemarié, F., Chelton, D., Illig, S., Hall, A., 2016b. Modulation of wind work by oceanic current interaction with the atmosphere. Journal of Physical Oceanography 46(6), 1685-1704.

Rooth, C., Xie, L., 1992. Air-sea boundary layer dynamics in the presence of mesoscale surface currents. Journal of Geophysical Research: Oceans 97(C9), 14431-14438.

Seo, H., Miller, A.J., Norris, J.R., 2016. Eddy-wind interaction in the california current system: Dynamics and impacts. Journal of Physical Oceanography 46(2), 439-459.

Vancoppenolle, M., Bouillon, S., Fichefet, T., Goosse, H., Lecomte, O., Morales Maqueda, M., Madec, G., 2012. The louvain-la-neuve sea ice model. Notes du pole de modélisation, Institut Pierre-Simon Laplace (IPSL), Paris, France (31).

$\mathrm{Xu}$, Y., Scott, R.B., 2008. Subtleties in forcing eddy resolving ocean models with satellite wind data. Ocean Modelling 20(3), 240-251. 